\title{
Co-constructing a Conceptual Understanding of System Enactment
}

\author{
Cathleen M. Morey ${ }^{1}$ (1) \\ Accepted: 3 December 2021 / Published online: 13 January 2022 \\ (c) The Author(s), under exclusive licence to Springer Science+Business Media, LLC, part of Springer Nature 2021
}

\begin{abstract}
System enactments are co-created phenomena characterized by confounding and emotionally charged multi-person interactions that emerge through the convergence of patients' complex psychopathology, staff vulnerabilities, and the organizational dynamics of the clinical system in which all are embedded. There is ample literature about the psychoanalytic construct of enactment in the therapeutic dyad. Though systems-based clinicians often experience system enactments which transcend the dyad and occur within the projective field of the system, there is no comparable literature that discusses this phenomenon. This paper describes a qualitative study that investigated how psychodynamic clinicians understood the phenomenology and impact of system enactments on clinicians, treatment processes and organizational climate. Major themes were identified through qualitative analysis of the data. The following four key findings were distilled from the resulting themes of the study's two research questions: (1) Clinicians conceptualize system enactments from a classical perspective; (2) System enactments have an experiential impact on clinicians in the domains of affect, cognition, behavior, and physiological arousal, which may be related to secondary traumatic stress responses; (3) Clinicians demonstrate a collapse of mentalizing associated with ruptures in the patient's treatment, conflict in the working relationships between staff, and problematic organizational dynamics; and (4) Interconnected and reciprocal interactions among all levels of the system including patient subsystem, individual staff subsystem, intra-staff subsystem and organizational subsystem, are shaped by the impact of system enactments. A conceptual understanding of system enactmentis outlined, and implications for clinical social work education and practice, organizational policy-making and research are addressed.
\end{abstract}

Keywords Enactment $\cdot$ System $\cdot$ Organizational dynamics $\cdot$ Secondary traumatic stress $\cdot$ Mentalizing $\cdot$ Constant comparison method

Clinical social workers who practice in treatment systems, such as an intensive outpatient program, residential setting, or psychiatric hospital, work with complex patients who generally have at least several co-morbid psychiatric conditions, including but not limited to mood, personality, substance use and psychotic disorders, as well as trauma histories and suicidality. The socially co-constructed environment of a clinical system is comprised of patients and multiple interprofessional mental health practitioners. Both patients and clinicians embody and differentially hold the structure, mission and socio-cultural dynamics of the organization.

Theoretical frameworks that synthesize psychodynamic principles with a systems perspective are critical to guide clinical social work practice in these settings. The literature

Cathleen M. Morey

cathleen.morey@austenriggs.net

1 Austen Riggs Center, Stockbridge, MA 01262, USA offers a systems perspective of how some fundamental psychoanalytic constructs inherent to the therapeutic dyad such as splitting, transference and countertransference dynamics, and projective identification manifest as multi-person dynamics and processes in the projective field of a treatment system (Betan et al., 2005; Gabbard, 1989; Greene, 1993; Main, 1957; Menninger, 1998; Stanton \& Schwartz, 1954). Notably, the phenomenon of enactment, which is a fundamental psychoanalytic construct, has not been conceptualized from a systems perspective for application to clinical practice in systems.

Enactment in the therapeutic dyad is characterized by an unconscious repetition of conflicted material from the life history of both patient and therapist that unfolds in the therapeutic situation. Mutual projective identifications stimulate uncharacteristic verbal and non-verbal interactions between the two parties. (Maroda, 1998; Plakun, 1997, 2007). While the reflective capacity of the therapist is compromised, their 
interpretive function is supplanted with unmetabolized behavioral communication. A fictionalized case example illustrates the phenomenology of enactment within the patient-therapist dyad.

A 40-year-old cis-heterosexual, White identified woman, Grace, had been in three-times weekly psychotherapy for several years with an older White female identified therapist. Grace had experienced childhood relational trauma perpetrated by family members that included neglect, as well as emotional, physical and sexual abuse. Throughout adulthood, Grace repetitively re-enacted her relational trauma narrative through multiple sadomasochistic sexual encounters with men. Grace had been in a sadomasochistic relationship with a new partner, Theo for several months when he began to become possessive and controlling of her. Theo texted Grace constantly throughout the day, demanding to know her whereabouts at all time. He repeatedly accused her of lying and of having sex with other men-a co-worker, a neighbor, a waiter in a restaurant. In this context, a co-constructed enactment emerged in the therapeutic relationship. For several months the therapist listened to Grace recount repetitive stories about Theo's abusive behaviors, with little to no curiosity about what she was caught up in. The therapist felt helpless, useless and worse yet, complicit since she felt that she was bearing witness to chronic relational violence and abuse. Grace began to deteriorate, experiencing flashbacks, agitation, hyperarousal, difficulty sleeping, numbing, dysphoria, and difficulty functioning at work. Feeling inadequate and powerless to help Grace get hold of what she was in, the therapist began to detach and feel indifferent to her struggles, likely enacting the bystander role of Grace's neglectful mother. In contrast, the therapist was shocked and horrified after reading Theo's text where he called Grace "a slut, a whore and white trash." These dynamics "hooked" the therapist's vulnerabilities related to her own life history, which set the enactment into motion. The therapist grew up with a father who emotionally, verbally and physically abused her mother. As a young girl, the therapist felt helpless and powerless; she was unable to stand up to her powerful father to protect her mother. In the throes of an enactment, the therapist's reflective functioning collapsed and she forcefully instructed Grace to immediately send Theo a text telling him that she was ending their relationship. The therapist reacted by taking action to protect a vulnerable woman from an abusive man-something she had been unable to do as a child.

Understanding enactment requires distinguishing it from related constructs such as transference, countertransference, projective identification, parallel process, and the intersubjective concepts of 'doer/done to' dynamics (Benjamin, 2014). Relational and intersubjective perspectives emphasize that transference and countertransference dynamics are co-created by the patient and therapist (Mitchell 1988, 1993; Ogden, 1994). Transference phenomena reveal the patient's subjective experiences and search for developmentally new objects. Countertransference refers to feelings induced in the therapist, often as a result of projective identification. These feelings are stimulated by the patient, as well as by the therapist's own psychological state and history.

A contemporary formulation of projective identification poses that both patient and therapist unconsciously and mutually project disowned aspects of their self-experiences into the other in the co-created interpersonal field (Mitchell, 2014). Each party is induced to identify with and experience feelings that are congruent with the disavowed projective fantasies of the other. Projective identification becomes a precursor for enactment (Ringstrom, 2010) when the therapist is unable to manage, integrate and interpret the patient's disowned states and instead succumbs by reacting and taking action. Parallel process was originally conceptualized as a projective identification process occurring in the supervisory dyad that reflects aspects of the patient's emotional experience and/or specific dynamics present in the patient-therapist dyad (Eckstein and Wallerstein, 1958; Searles, 1955). A relational perspective suggests that parallel process is a complex, co-created and interactive process that is multidirectional in nature. It manifests within a relational field between and among the supervisor, supervisee, and patient/ family, and is influenced by the organizational and systems dynamics in which all are embedded (Shea, 2019). Parallel process, at times, but not always, leads to enactment (Mendelsohn, 2012).

Benjamin (2014) introduced the notion of intersubjective thirdness as an aspect of enactment. Her theory proposed an early form of thirdness, in which patient and therapist co-construct a shared space, called the 'one in the third' that involves affective resonance, union and accommodation. She referred to later forms of moral and symbolic thirdness that are marked by differentiation as the 'third in the one.' Benjamin posited that enactments emerge when the shared intersubjective third collapses and there is a breakdown in mutual recognition leading to complementary relations characterized by polarizing 'push-me/pull-you, doer/doneto' dynamics.

When treatment occurs in a system, the relational paradigm is multi-dimensional in nature with multiple subjectivities interacting in a mutually co-constructed space. In this context, systems-based clinicians frequently experience confounding and emotionally charged multi-person enactments that transcend the dyad and occur within the projective field 
of the system. This phenomenon is referred to as a system enactment. A clinical example illustrates this phenomenon.

Quinn, a young adult transgendered male to female, who identified as White and as affiliated to a particular religious denomination, was admitted to an all-female residential treatment program of that religious affiliation. The organization had not previously treated transgendered individuals. The clinicians voiced their concerns to upper management about having no established policies, organizational structure, or clinical protocols to work with transgendered clients. In turn, administration ignored their concerns and gave them the directive to admit the client. Quinn felt unsafe in the setting and expressed, both verbally and through behavioral actions, her experiences of bias and prejudice from staff and other clients. For example, Quinn became reactive when a staff person led her to a bathroom labeled "male," while the staff member disavowed this action as having any meaning. Moreover, based on their religious beliefs, some of the staff on the team were highly 'offended,' very upset, and biased towards transgendered individuals, which created a split on the team and in staff meetings. In this context, Quinn's self-harming behaviors began to escalate. Staff felt Quinn was testing the limits in ways that were unsafe and a decision was made to administratively discharge her due to her lack of compliance with the program's safety policies.

As this example illustrates, a system enactment is an actualization of unconscious, mutual and complementary projective identification processes that are co-constructed through a convergence of the patients' complex psychopathology, staff vulnerabilities, and the organizational dynamics of the clinical system in which all are immersed. These types of enactments are associated with treatment ruptures, frayed working relationships among staff, and problematic organizational dynamics. Unaddressed system enactments can have negative impacts on treatment processes, including treatment ruptures, therapeutic impasses, and treatment resistance, as well as premature or precipitous terminations of treatment (Morey, 2019). In the context of these enactments, clinicians experience strain and conflicts in their interpersonal working relationships that affect their clinical work. Organizational health is compromised as well by unrecognized and unresolved system enactments that are fueled by structural and social dynamics within the system. When patients experience failed treatments associated with unresolved system enactments, emotional, social and financial costs are incurred.

The purpose of this paper is to summarize a qualitative study that examined the co-constructed phenomenon of system enactment from the perspectives of twenty-two clinicians who held psychodynamic theoretical orientations and practiced in treatment systems across the United States. The goal of this study was to develop a conceptual understanding of system enactment. Research questions included: (1) How do psychodynamic clinicians practicing in treatment systems conceptualize the phenomenon of enactment that occurs among multiple parties involved in the treatment task? (2) How do these clinicians understand the impact of this phenomenon on clinicians, treatment processes and organizational climate? A synopsis of the methodology is outlined and major themes were identified through qualitative data analysis. A conceptual understanding of system enactment is offered that was formulated through a synthesis of the literature and a review of the four key findings. The strengths and limitations of the study are considered, as are implications for clinical social work education and practice, organizational policy-making, and directions for future research.

\section{Central Terms and Concepts}

\section{Enactment in the Therapeutic Dyad}

The notion of enactment is almost exclusively defined in the literature as a dynamic process that happens between patient and therapist in individual treatment. Early scholarship conceptualized enactment from a classical perspective that was based on a one-person psychology. Enactments that emerged between patient and therapist were conceived of as emanating solely from the intrapsychic conflicts of the patient (Jacobs, 1986; Sandler, 1976). Post-classical formulations shifted to a two-person psychology of enactment in which the subjectivities and intrapsychic vulnerabilities of both the patient and the therapist converge to co-create enactments (Bass, 2003; Benjamin, 2014; Bromberg, 2011; Chused, 2003; Maroda, 1998 and Plakun, 1997).

\section{Stress, Trauma, and Enactment}

Patients who require care in treatment systems have high psychiatry acuity and co-morbidity that often includes complex trauma histories and suicidality. Clinical work with this population can induce physiological stress responses and secondary traumatic stress in staff that are often accompanied by difficulties with mentalization. The neurobiology of stress is important to consider when thinking about enactments that emerge in these treatment settings (McEwen, 2007). A normal stress hormone response activates the autonomic nervous system, the hypothalamic-pituitary-adrenal axis, and the "fight-flightfreeze' response. According to McEwen, "stress hormones, such as cortisol, are involved in psychopathology, 
reflecting emotional arousal and psychic disorganization" (p. 891). McEwen's research about stress is applicable not only to patients with severe psychopathology, but also to clinical staff who experience traumatic stress in response to working with traumatized and suicidal patients. Trauma that is of a severe or chronic nature causes neurobiological dysregulation characterized by three main symptom clusters-hyperarousal, intrusion, and avoidance-resulting in cycles of activation and numbing (Cozolino, 2017). As staff experience dysregulated stress, which is an inherent neurobiological aspect of enactment, they may respond with sympathetic hyperarousal, characterized by emotional and physiological reactivity, or parasympathetic hypoarousal marked by numbing, affective detachment, and at times dissociation (Schore, 2012). According to Figley (1995), symptoms of secondary traumatic stress are activated in staff in the context of heightened affective moments of enactment.

\section{Mentalization}

Mentalizing capacities of clinicians are often affected as an element of system enactment. As a form of social cognition, Fonagy and Bateman (2006) refer to mentalization as the capacity "to understand actions by both other people and oneself in terms of thoughts, feelings, wishes and desires “(p. 3). Mentalizing within a treatment system is challenging because it requires maintaining an open, curious and reflective stance towards the mental states of multiple mindspatients and colleagues. Fonagy and Bateman (2006) discuss the notion of "mentalizing the system" and assert the importance of including mentalizing systemic approaches such as "mentalizing supervision" and a "mentalizing team."

\section{Multi-person Clinical Phenomena in Treatment Systems}

Several authors examine how clinical phenomena related to enactment, such as splitting, "acting out," countertransference and reenactment unfold in the multi-person context of a treatment system (Krikorian \& Fowler, 2011; Monroe et al., 1995; Skogstad, 2006). These studies describe how the object relational paradigms of patients evoke split countertransference reactions in treatment team members, who in turn enact these dynamics. From this classical perspective, patients are viewed as inducing staff into problematic interactions by recreating their intrapsychic object relations in the interpersonal relationships among team members. The subjectivities of multiple staff members and the organizational dynamics of the system that contribute to the emergence of such phenomena are not considered.

\section{Systems Theory}

Systems theory proposes that a system is an entity comprised of interrelated and interdependent parts, known as subsystems that interact in a fluid, non-linear and multi-directional manner to form a 'whole,' whose characteristics are different from the individual parts (von Bertalanffy 1969; Bateson, 1979). The component and interactive subsystems - the individual, group and organization-interact to form the total system (Czander, 1993).

System enactments take shape and unfold among these subsystems, which are correlated with the following realms: intrapsychic, interpersonal and organizational. The system is viewed as having its own life created by conscious and unconscious interacting subjectivities among various subsystems in an intersubjective field that constitute one another in a mutual and reciprocal manner (Czander, 1993; Miller, 1993).

\section{Organizational Context}

Organizations have both structural and social components. The structural aspects of an organization encompass the "day-to-day workings of the system," (Shapiro, 2011) such as hiring and staffing practices, policies, procedures, roles and division of labor. In contrast, the social aspect of an organization is constituted by a complex, competitive and political system of interprofessional staff and patients who relate to the organization itself. Staff and patients embody and enact the ideology, socio-cultural values, and mission of the organization, as well as social dynamics such as hierarchy, power, privilege, oppression and racism (Eisenberg, 1997; Herman et al., 2002; Salhani \& Coulter, 2009; Stapley, 2001). Thus, a formulation of system enactment that synthesizes intrapsychic, interpersonal and organizational dynamics, posits that this phenomenon isco-constructed through a multi-directional and intersubjective flow of dynamic processes. On an intrapsychic level, patients and staff embody, and enact in the interpersonal arena-the structural and social dynamics of the organization in a nonlinear, interconnected and reciprocal manner.

\section{Methods}

A qualitative approach, employing the constant comparison method (Anastas, 1999; Glaser \& Strauss, 1967; Strauss \& Corbin, 1990, 1998), was used in this study to explore the subjective meaning of system enactment from the study participants' lived experiences and knowledge of reality (Charmaz, 2006). A social constructivist epistemological perspective undergirds this study, which assumes no ultimate truth and privileges the notion that 
social experience is contextually bound (Anastas, 2012). The study aimed to describe and capture the ways that psychodynamic clinicians practicing in treatment systems (1) conceptualize the phenomenon of enactment that occurs among multiple parties involved in the treatment task and (2) understand the impact of this phenomenon on clinicians, treatment processes and organizational climate. This study was approved by the Ethics Committee of Smith College School for Social Work.

\section{Three-phase Methodology Plan}

A three-phase methodology plan was utilized. In the first phase, a provisional conceptualization of system enactment was developed based on a review of the existing literature, and my clinical practice experience working in treatment systems. The second phase involved consultation with nine clinical experts to elicit their feedback, which enhanced the credibility of the conceptualization (Denzin $\&$ Lincoln, 2011). Based on their input, the provisional definition was clarified and revised to formulate a working conceptualization of system enactment. The working conceptualization postulated that there were three subsystems-intrapsychic, interpersonal, and organizationalalong with seven prominent features of system enactments, including: (1) uncharacteristic affect; (2) loss of reflective functioning; (3) reactivity or withdrawal; (4) uncharacteristic verbal or non-verbal interactions; (5) deviation from therapeutic frame; (6) polarization dynamics; and (7) emergence of treatment ruptures. Phase three involved utilizing the working conceptualization to conduct in-depth semi-structured interviews with a non-probability, purposive sample of 22 clinicians. There were two inclusion criteria for respondents: (1) knowledge of psychodynamic and systems theory, and (2) at least two years of clinical experience working in a treatment system defined as intensive outpatient treatment program, a residential setting or a hospital. Two exclusion criteria were delineated for this sample, including clinicians who worked in my treatment system and the nine clinical experts who were interviewed in the conceptualization process.

In advance of the interview, the working conceptualization of system enactment was emailed to participants and they were asked to be prepared to discuss a system enactment from their clinical practice. An open-ended interview guide with semi-structured questions (See "Appendix A") was used to elicit a narrative flow to explore their observations, perspectives and understanding of the phenomenology of system enactment, and the impact on clinicians, treatment processes and organizational climate. Through an iterative process, the interview guide was modified during the process of data collection (Charmaz, 2006).

\section{Sample}

The sample was predominately white, female clinical social workers with a mean age of 50 years old, and a mean of 16 years working in treatment systems. The participants were almost evenly distributed across the three clinical settings of interest-intensive outpatient programs, residential settings, and hospitals. All respondents described their patient population as having complex and co-morbid psychopathology.

\section{Data Analysis}

The interview data were manually coded and analyzed, guided by the principles of the constant comparison method (Anastas, 1999; Glaser \& Strauss, 1967; Strauss \& Corbin, 1990, 1998). Data analysis was initiated by reading and scrutinizing each transcribed interview. Data collection, sampling and analysis occurred concurrently. In this iterative procedure, newly gathered data were continually compared with previously collected data, which enabled new questions to be posed, and emerging categories and ideas to be tested in subsequent interviews. The method facilitated a fourway comparison of codes: (1) across data sets, (2) in relation to the literature, (3) in comparison to my own clinical experience, and (4) in relation to the provisional definition of system enactment. During data analysis, two strategies were utilized to develop awareness of and reduce researcher bias (Anastas, 1999). First, I developed an audit trail which detailed my research procedures, including my notes from the field, interviews, and coding processes. Reflexivity was used throughout the process. While sharing this audit trail information with a senior researcher, we discussed the data with a focus on potential personal and/or theoretical research bias. In addition, I used a second coder to read and code two full transcripts. The second coder was a clinical social work $\mathrm{PhD}$ candidate with a strong background in research, however lacking clinical practice experience working in treatment systems. This omission proved to be an asset since it helped to mitigate personal and theoretical biases. To minimize possible bias, I directed my second coder to examine the transcribed interviews for evidence of participant or researcher bias. We also engaged in several peer debriefing sessions (Guba \& Lincoln, 1989; Lincoln \& Guba, 1985), where my second coder asked probing questions and suggested alternative ways of thinking about analyzing the data.

\section{Results}

The results of this qualitative analysis will be presented through a discussion of the major themes that were extracted from the data in response to the study's two focal research questions. 
Table 1 Experiential descriptors and impact of system enactment on clinicians

\begin{tabular}{ll}
\hline Domains & Most common responses \\
\hline Affective dysregulation & Amplified affect \\
$\begin{array}{c}\text { Cognitive disequilibrium } \\
\begin{array}{c}\text { Uncharacteristic verbal or } \\
\text { non-verbal behaviors }\end{array}\end{array}$ & Loss of reflective functioning \\
\begin{tabular}{c} 
Somatic manifestations \\
\hline
\end{tabular} & Moderate to severe physiological arousal \\
\hline
\end{tabular}

\section{Themes from Research Question 1: Phenomenology of System Enactment}

The first research question explored how psychodynamic clinicians working in treatment systems conceptualize the phenomenon of enactment. The following six themes emerged from the data: (1) experiential descriptors and impact of system enactment on clinicians, (2) trauma dynamics of patients, staff and organization, (3) beyond the dyad, (4) projective field of the treatment system, (5) sociocultural context, and (6) conceptual ideas about the phenomenon.

The first theme highlighted that all of the participants described system enactment in experiential self-referential terms. They shared being affected in four domains. The following Table 1 delineates these four domains and captures the most common responses clinicians had when activated in these domains.

The second theme linked the trauma dynamics of patients, staff and the organization to system enactments. All respondents reported working with patients who had trauma histories and described how the trauma dynamics of their patients were associated with system enactments. More than half of the respondents reported that system enactments were associated with clinician experiences of secondary traumatic stress, while half of the respondents spoke about the relationship between organizational trauma dynamics and system enactments. The third theme emphasized that system enactments occur among multiple participants—beyond the therapist/patient dyad. All respondents described system enactments as involving dynamic processes among multiple participants, including patients, families, treatment team members, supervisors, administrators, CEOs, members of the institution's Board of Directors, and parent companies. Many respondents gave examples of treatment team members being involved in system enactments. The fourth theme focused on the projective field of the treatment system in which system enactments occur. Over half of the respondents reported that treatment team meetings were a prime setting for the emergence of this phenomenon. The unit/milieu was another common setting where these enactments occurred. In theme five, more than half of the respondents discussed how sociocultural dimensions, such as race, religion, economic factors, gender identity, sexism and patriarchy were operative in system enactments. The sixth theme outlined four ideas reported by respondents about their conceptual understanding of system enactment, which included: (1) initial formulation, (2) evolved formulation and clinician's subjectivity, (3) clinician's objectivity, and (4) clinical utility. Most respondents described a classical formulation of system enactment. Over the course of the interviews, those who initially led with a classical formulation went on to discuss evolved formulations that included conceptualizing the phenomenon from a relational or intersubjective postclassical perspective. Several respondents discussed the loss of clinical objectivity as part of the experience of being in a system enactment, and more than half of the respondents shared their view that system enactments have significant clinical utility.

\section{Themes from Research Question 2: Impact of System Enactment}

The second research question explored the impact of system enactment on clinicians, treatment processes and organizational climate. Each of the four themes identified from the data will be presented and summarized.

The first theme highlighted the various precipitants to system enactments. Examples include the business function of the organization superseding and resulting in deviations from standard clinical practices, a harsh organizational climate, and factors of power and influence affecting clinical decision making, to name a few. These influences powerfully affected individual clinicians in the following four domains: (1) affective dysregulation, (2) cognitive disequilibrium, (3) uncharacteristic verbal and/or non-verbal behaviors and (4) somatic manifestations, which in turn significantly affected the treatment of the patient. Respondents were able to make productive therapeutic use of system enactments when they utilized supervision and consultation, relied upon the therapeutic use of self, held a systems perspective, and maintained a framework to conceptualize system enactments. They reported positive effects of engaging system enactments such as increased sense of clinical competency, enhanced trust and safety with colleagues, and positive morale. In contrast, negative impacts included a lack of safety and trust with colleagues and administrators, no time or space for reflective clinical practice, and a lack of clinical leaders to provide an integrative function. Respondents shared that unresolved system enactments had powerful negative effects on them including decreased sense of clinical competency, low morale, thoughts of quitting and actively seeking other employment, and moderate to severe somatic manifestations that appeared to be linked to their exposure to direct or indirect traumatization. 
The second theme emphasized the impact of system enactments on interpersonal dynamics among treatment staff. Respondents described a similar array of conditions that might catalyze a system enactment as reported in theme one. However, they reported that the system mobilized around the precipitating issue in a way that most powerfully affected intra-staff functioning, leading to overt or covert polarization dynamics, which in turn significantly affected the treatment of patients. Facilitative conditions included provision of an organizational holding environment, culture, and shared theoretical framework to engage and repair intra-staff tensions and conflict. Working through intrastaff dynamics led to positive outcomes such as increased staff cohesion, improved intra-staff working alliances, and enhanced trust, safety and interdependency among colleagues. In contrast, constraining conditions involved intrastaff dynamics such as competition, lack of trust, respect and value, and experiences of oppression, hierarchy, and power differentials. Respondents shared that under these conditions, the treatment of patients became almost peripheral because the primary focus of the staff is on their own interpersonal tension and conflict with each other. Moreover, they shared that at times, such interpersonal dynamics led to a breakdown of intra-staff functioning, poor morale, destruction of collegial relationships and a collapse of clinical services.

The third theme outlined how unaddressed tensions in the staff subsystem related to problematic organizational dynamics converged with patient dynamics to precipitate system enactments. Respondents reported that such enactments emerge along the organization's preexisting fault lines, activating divisive but unspoken fractures in the staff subsystem, such as conflicts between clinicians and administrators, junior and senior staff, and along gender lines to name a few. Such dynamics not only negatively affected the treatment of the patient, but also negatively affected the staff's relationship to the organization. Facilitative conditions to productively engage organizational dynamics associated with system enactments included utilizing clinical staff meetings as a reflective space for staff to process divisive organizational issues, and staff holding a shared perspective about the clinical utility of enactments. Working through a process of rupture and repair not only with patients, but among staff and at an organizational level led to increased morale and feelings of empowerment, which in turn improved the organizational health of the system. Conversely, respondents reported a range of constraining conditions and negative consequences related to organizational dynamics. These included a lack of structure and resources to address system enactments, a culture of hostility and blame, a loss of connection to the mission, a rupture in their relationship to the organization, staff turnover, and at times, the organization closing.
The fourth theme focused on the impact of system enactments on patients' treatment. As outlined in the previous three themes, the treatment of the patient is always affected however, in this theme, respondents brought attention to the severity and at times, egregiousness of a system enactment's effect on the patient. These enactments emerged when constraining conditions superseded facilitative factors. Respondents reported widespread constraining conditions such as problematic organizational structures, breakdown in staff communication, perceptions of inequity among staff, experiences of oppression due to organizational hierarchy, harsh and blaming organizational culture contributing to staff feelings of loss of control, disempowerment, and lack of safety. Additionally, there was an absence of a systems perspective, no clinical framework for rupture and repair, and no shared reflective space in the system for staff to work through these enactments. In this context, respondents reported a range of severe effects on patients' treatment such as premature and administrative discharges, boundary violations by staff, patients being labeled "treatment resistant," and in some instances staff violating patients' rights.

In exploring whether there were any relationships among these four themes, what emerged was the strong interconnection of the impact of system enactment among the four levels of the system: patient subsystem, individual staff subsystem, intra-staff subsystem and organizational subsystem. Though clinicians described the concurrent impact of system enactments on all levels of the system, they shared their subjective experiences of being affected more powerfully in one subsystem versus another, depending on their role and their involvement in the system enactment. A case example illuminates the complexity of a system enactment.

A 14-year-old, White, gender non-conforming patient, Teagan, was 'stuck' on the adolescent unit of a psychiatric hospital due to conflict between the clinical staff, Teagan's parents and the Department of Social Services. Staff experienced a harsh organizational culture in which management blamed them for adverse patient outcomes. Problematic structural differences and perceptions of inequality existed between the unit's primary treatment team of master's educated clinicians and the second shift direct care staff, who had less education and training, but spent the most time with patients. As Teagan experienced a loss of control over their extended length of stay on the unit, they manifested aggressive behaviors, which the direct care staff experienced as threatening. Tension and conflict began to escalate between the primary treatment team and the direct care staff, manifested by a breakdown in communication. Direct care staff felt directed by the primary treatment team about how to intervene with Teagan and in the process, their authority and autonomy 
were eclipsed. They experienced a loss of control and felt oppressed by the organizational hierarchy. A system enactment emerged as direct care staff responded to Teagan's aggressive behaviors with harsh, restrictive interventions rather than relational engagement. Direct care staff exercised control with Teagan through an increased number of physical restraints, which could be viewed as an enactment of their anger towards the primary treatment team and the organization. In turn, Teagan escalated with self-harming behaviors and made a suicidal gesture. The direct care staff egregiously responded to this gesture by violating Teagan's patient rights. Shortly after, Teagan was precipitously discharged without a comprehensive disposition. In addition, an organizational rupture ensued between the direct care staff, the primary treatment team, and the clinical administrators of the hospital. This rupture resulted in ongoing sub-optimal and/or adverse patient outcomes, breakdown of intra-staff functioning, poor morale, clinician burnout and high staff attrition rates.

This vignette portrays how problematic structural and social dynamics of an organizational culture that was characterized by power struggles, inequalities and oppressive dynamics were enacted by staff and attributed to the psychopathology of the patient. The clinicians demonstrated affective, cognitive, behavioral, and physiological reactivity and dysregulation. They experienced a collapse of mentalizing in the context of intra-staff conflict and problematic organizational dynamics, which in turn was associated with a treatment rupture and an adverse patient outcome. This vignette vividly illuminates the concurrent impact of a system enactment on all four levels of the system: patient, individual clinician, intra-staff and organizational subsystems.

\section{Discussion}

Systems-based clinical social work practice requires knowledge of treating patients with complex psychiatric conditions, interprofessional collaboration skills, an awareness of how clinical phenomena manifest as multi-person dynamics in systems, and an understanding of organizational dynamics. Given the prevalence of systems-based clinical work, it is imperative that psychodynamic principles are conceptualized from a systems perspective to facilitate theory-informed clinical social work practice. Yet, social workers may be limited by psychodynamic theories and clinical approaches that have not been developed and expanded in scope beyond application in the therapeutic dyad to application at a systems level. The purpose of this study was to investigate the phenomenology and impact of system enactment from the perspective of psychodynamic clinicians who practice in treatment systems to develop a conceptual understanding of this phenomenon. In considering the resulting themes of the two focal research questions of this study, this research has distilled four key findings around which the conceptual understanding of system enactment will be presented and discussed.

\section{Key Findings}

\section{Classical and Post-classical Formulations}

The first finding was that the majority of clinicians initially conceptualized system enactment from a classical theoretical perspective. This finding is consistent with studies in the existing literature, which attribute problematic dynamics and interactions that occur in the multi-person context of treatment systems to the psychopathology of the patient. Respondents in this study were likely sensitized to conceptualize from a one-person viewpoint due to their exposure to the extant literature about these systems phenomena, which is limited to oneperson perspectives. Reliance of respondents on a one-person perspective at the onset of interviews could also be viewed as a defense against reflecting on and exposing their own unconscious processes and vulnerabilities that contribute to these types of enactments. Ascribing to a one-person psychological framework potentially relieved the respondents of guilt, shame and other painful feelings associated with their participation in the enactment. Upon discussing their clinical vignettes, the majority of respondents shifted to a two-person formulation as they shared how their own vulnerabilities and subjectivities were mobilized in system enactments. One hypothesis about a shift from a classical to a post-classical explanation of system enactment is that as respondents described the relational aspects of their own clinical work with depth and nuance, they were more able to acknowledge how their own subjectivities influenced and shaped system enactments. This finding expands the literature on enactments in multi-person treatment systems by providing a contemporary relational perspective in which staff vulnerabilities and subjectivities are considered as equally relevant in a system enactment as those of the patient.

\section{Experiential Impact on Clinicians and Secondary Traumatic Stress}

The second finding of this study was the association between system enactments, trauma dynamics of patients, and stress responses experienced by staff. Patients who require care in treatment systems often have a high prevalence of trauma. Not surprisingly, all respondents endorsed working with patients who had trauma histories. They reported a link between the trauma dynamics of patient sand system enactments. The majority of respondents reported that system 
enactments affected them in four domains: affect, cognition, behavior, and physiological arousal. Respondents described dysregulation in these four experiential realms that manifested along a continuum from dysregulated stress to secondary traumatic stress responses.

This finding adds more descriptive depth and complexity to the prior literature about the physiology and neurobiology of stress, trauma, and enactments (Ginot, 2007; Schore, 2012; McEwen, 2007; Figley, 1995; Cozolino, 2017; Siegel, 2003, 2007; van der Kolk, 2014). Most respondents were involved in system enactments over a period of time-from weeks to months-during which they experienced prolonged and intense stress. Given that these enactments are of long duration, it is likely that clinicians experienced what McEwen (2007) describes as chronic stress characterized by the dysregulation of cortisol and other chemical mediators that are associated with emotional arousal and psychic disorganization. As respondents experienced dysregulated stress, they either responded with sympathetic hyperarousal, characterized by affective, behavioral and physiological reactivity, or parasympathetic hypoarousal marked by affective detachment, behavioral withdrawal and physiological numbing. In both states, cognitive functioning was altered, notably by a collapse of mentalizing.

Respondents drew attention to the impact that system enactments had on their behaviors and cognition. This finding diverges from this literature, by suggesting that system enactments occur when the neurobiological effects of dysregulated and secondary traumatic stress push clinicians beyond their window of tolerance, leading to cognitive and behavioral dysregulation, in addition to affective and physiological hyperarousal and hypoarousal. The majority of respondents described experiencing shifts in their cognitive functioning such as rumination, self-critical thoughts, intellectualization, group think, fragmented and disorganized thinking. All respondents described speaking in unusual ways or behaving in ways that were uncharacteristic for their clinical practice, including engaging in divisive polarization and splitting dynamics with their colleagues, and taking actions that deviated from the therapeutic frame. In another divergence, findings from this study did not fully support the literature that described how enactments contribute to clinicians' experience of vicarious traumatization. Respondents did not endorse the full range of specific criteria that are often identified with vicarious traumatization such as dramatic transformations in their sense of self, beliefs and/ or worldview (Pearlman and Saakvitne, 1995).

The second finding may appear to support a classical formulation, in that the trauma dynamics of patients are viewed as stimulating traumatic stress responses in clinicians. Though this is a reasonable inference, it does not take into account a contemporary, postclassical perspective, endorsed by many respondents, wherein system enactments emanate from a multi-directional and intersubjective flow of dynamic processes between patients and staff, along with the organizational context in which they are embedded. Such a viewpoint considers a cyclical pattern whereby dynamics, which may or may not be directly connected to the patient, such as sequelae related to staff experiences of secondary traumatic stress, also precipitate, influence and shape system enactments.

\section{Mentalization}

The third finding was that when clinicians were in the throes of a system enactment, many reported experiencing cognitive disequilibrium, most often marked by a collapse of mentalizing. Overwhelming affect, stress and trauma can lead to a breakdown in mentalizing, which often manifests as a non-reflective, non-curious stance about the feelings and thoughts of others (Asen \& Fonagy, 2012). In the context of system enactments, many respondents experienced disturbances in their mentalizing capacity. In these nonmentalizing states, they struggled to hold onto their clinical minds and experienced difficulty holding the mental states of others in mind. Furthermore, patients with complex psychiatric co-morbidity generally have low levels of reflective functioning and thus their difficulties with mentalization create challenges in how they interact with treatment staff. Respondents described the importance of creating and utilizing individual reflective spaces, such as individual supervision and consultation, as well as shared reflective spaces, including team meetings and clinical staff meetings. This finding adds complexity to the literature about mentalizing and systems.

\section{Interconnected and Reciprocal Interactions Among Four Subsystems}

The fourth finding of this study suggested that system enactments involve multiple participants and are precipitated in a number of different ways in various spheres of the treatment system. This phenomenon is co-constructed through mutually, reciprocal subjective interactions that are non-linear in nature. Indeed, this finding illustrates that there is a powerful interrelationship of the impact of system enactment among the system's four interactive levels. These levels include the patient, the individual clinician, the intra-staff, and the organizational subsystems. Based on a review of the literature, it made sense to initially conceptualize the system as being comprised of three interrelated subsystems-the individual, group and organization - that interact to form the total system. I hypothesized that system enactments took shape and unfolded in these three subsystems, which were correlated with three domains: intrapsychic, interpersonal and organizational. I conceived that system enactments 
engaged (1) intrapsychic vulnerabilities of both patients and staff; (2) interpersonal processes between patients and staff, and among multiple staff; and (3) organizational dynamics. This study delineated four interactive subsystems that diverged from the literature on systems in two ways. First, respondents' subjective descriptions differentiated the intrapsychic domain into two distinct subsystems-that of the patient and that of individual clinicians. And second, respondents did not endorse a patient/staff subsystem, rather they described an intra-staff subsystem.

\section{Conceptual Understanding of System Enactment}

A conceptual understanding of system enactment was formulated through a synthesis of the literature and this study's key findings which were distilled from the resulting themes of the two research questions. All participants talked about system enactments as co-constructed phenomena that are shaped by mutual and complementary projective identification processes between the four levels of a system that are all embedded in a sociocultural context. These levels include the patient subsystem, the individual clinician subsystem, and the intra-staff subsystem that reflects interactions among staff, as well as the organizational subsystem that reflects dynamics which emerge among individuals in relation to the organization's culture, policies, procedures, structure and social dynamics. System enactments have an affective, cognitive, behavioral and physiological impact on clinicians, and are associated with dysregulated stress and secondary traumatic stress responses. As a system enactment unfolds, staff may respond with sympathetic hyperarousal or parasympathetic hypoarousal. In both states, cognitive functioning is altered, notably by a collapse of mentalizing. In this non-mentalizing state, staff vulnerabilities and subjectivities are mobilized and they engage in overt or covert polarization dynamics that are characterized by ruptures in the patient's treatment, splits and frayed working relationships among staff, and problematic organizational dynamics. Thus, seven features are generally prominent when a system enactment is operative: (1) affective dysregulation; (2) cognitive disequilibrium; (3) uncharacteristic verbal or non-verbal interactions; (4) somatic manifestations; (5) deviation from the therapeutic frame; (6) polarization dynamics among staff; and (7) the emergence of treatment ruptures.

\section{Strengths and Limitations}

This qualitative study has several strengths. Access to professional networks of clinicians working in treatment systems was an asset of the study, which not only facilitated recruitment of eligible participants, but also the quality of the interviews. Participants had substantial clinical experience working in treatment systems. In addition, the sample was almost evenly distributed among the three clinical settings of interest in this study-intensive outpatient programs, residential facilities, and hospitals. Thus, the findings may be considered representative of respondents' experiences of system enactments across a range of settings.

Several limitations of this study are important to consider. It was difficult to recruit this sample. This challenge in recruiting systems-based clinicians with psychodynamic knowledge may be reflective of the shifting nature of practice contexts. The study's small sample size is a limitation because it did not allow for much variability across demographic and descriptive factors. In particular, the majority of participants identified as white, female clinical social workers. It is reasonable to hypothesize that the social identities of participants are linked to their experiences and understanding of system enactment-a socially co-constructed phenomenon. The study did not include a question related to the influences of social location and sociocultural factors on system enactments. This limited an examination of how these factors shaped understanding of system enactments. Another potential limitation of this study is that only clinicians who held psychodynamic theoretical orientations and systems perspectives were interviewed. Utilizing this selection criterion allowed for more focused findings, but also limited the development of a conceptual understanding of system enactment to the ideas, perceptions and experiences of this subgroup of clinicians who shared the same theoretical and clinical practice orientation.

\section{Implications for Clinical Social Work Education and Practice, Organizational Policy-Making, and Research}

Clinical social workers are educated to be systems practitioners; they hold and articulate a systems perspective underpinned by the "person in environment" construct (Kondrat, 2013). From this standpoint, they are ideally suited to recognize and engage system enactments. The conceptual understanding of system enactment developed from this study can help educate clinical social workers, and other mental health practitioners to recognize, understand, and engage this phenomenon, which they will likely encounter given its ubiquitous nature. Specific recommendations for clinical social work education, direct practice, organizational policymaking, and directions for future research are suggested by the study's findings. First, it is important for organizations to promote a psychodynamic systems treatment approach that is grounded in a psychoanalytic understanding of individuals and systems. Second, it is necessary to negotiate and create an organizational culture that supports clinical social workers to work effectively with system enactments. To do so requires the provision of a holding environment that promotes curiosity, and attenuates guilt and shame in staff 
when they are involved in a system enactment. In this way, staff can develop a clinical practice of exploring and discussing the possibility of a system enactment at all times given the prevalence of this phenomenon. Through this practice, system enactments may be recognized earlier and engaged productively before they lead to detrimental outcomes.

Adequate organizational infrastructure is needed to provide staff with opportunities for reflective clinical practice given that a collapse of mentalizing is a hallmark of system enactment. Maintaining and expanding the mentalizing capacities of clinical social workers requires individual supervision, peer consultation, team meetings, and broader clinical staff discussions that are rooted in a conceptual understanding of system enactment. Utilizing a psychodynamic systems approach allows staff, who are not directly involved in a system enactment to provide consultation and support to those clinicians who are directly involved. In this way, case formulations about what is being enacted at a systems level can be developed. This process helps to restore mentalizing capacities as clinicians discuss, process and work through system enactments. Such organizational provisions require time, resources, and money. Yet, organizational provisions and clinical protocols that promote reflective practice might mitigate ruptures in the treatment of patients that often result in premature and administrative discharges, and might potentially alleviate clinician burnout, high attrition rates and employee turnover. And finally, it is recommended that staff reflect on the 'fault lines' of the organization at any given time to develop awareness of how particular organizational factors, such as financial strains, staffing shortages, recent patient suicide, and COVID-19 related stressors, to name a few, may contribute to system enactments.

This study generated several directions for future research. First, a study that explored the perspectives of multiple staff involved in a system enactment could further understanding of this complicated dynamic. Another area for research involves examining the influences of staff and patients' intersecting social identities and sociocultural factors on system enactments. Third, a study aimed at investigating how clinicians utilize formulations of system enactments to inform their interventions could provide valuable practice data and knowledge.

\section{Conclusion}

Clinical social work practice in treatment systems, involving multiple participants embedded in a complex relational matrix, can be confusing and challenging. This study presents a systems level construct of enactment, with relational and psychodynamic underpinnings, termed system enactment. The conceptual understanding developed from the findings of this research contributes to the theoretical and empirical literature by offering clinicians a way to understand, recognize and productively engage a commonly encountered therapeutic phenomenon. Effectively engaging system enactments provides opportunities for clinicians to repair treatment ruptures with patients and to restore their working alliances with other staff. Furthermore, this conceptualization is congruent with a strengths-based approach inherent to clinical social work practice because treatment difficulties are conceptualized as co-constructed system dynamics, rather than dilemmas attributed exclusively to the psychopathology of the patient. The co-constructed conceptual understanding of system enactment developed through this study fills a gap in the theory and practice literature, and provides new empirical knowledge for clinical social work practice in systems that might potentially enhance clinical practice, organizational health as well as clinician health and well-being.

\section{Appendix A}

\section{Interview Guide}

(1) Have you experienced this type of phenomenon in your clinical work?

a. If so, what does it look like and how does it feel?

b. What are some indicators that tell you that you are experiencing this phenomenon?

c. How do you gain awareness when you are involved in a system enactment?

d. What are the kinds of terms you use to describe it?

e. When you encounter this phenomenon, how do you explain it to your colleagues?

f. Can you describe a clinical vignette from your practice experience that illustrates a system enactment?

(2) What is your theoretical framework to understand this phenomenon?

a. What theories do you consider to guide your clinical practice when you experience this phenomenon?

b. How is your conceptualization of this phenomenon similar or different from the conceptual definition I provided for you?

c. How do you see and understand the ways in which a system enactment is shaped by the intersection of 
intrapsychic, interpersonal and organizational factors of the system?

d. How do you think trauma experiences of patients, staff, and the organization may inform system enactments?

e. Have you experienced physiological/somatic responses as a result of being involved in a system enactment?

(3) Do you think system enactments impact treatment processes and organizational climate?

a. If so, how?

b. Do you use your understanding of system enactments to inform your clinical interventions? If so, please explain.

Acknowledgements The author wishes to acknowledge and thank Kathryn Basham, PhD, LICSW and Joanne Corbin, PhD for their generous and valuable feedback on this manuscript.

\section{Declarations}

Conflict of interest The author declares that the author has no conflict of interest.

Ethical Approval This study was performed in line with the principles of the Declaration of Helsinki and its later amendments. Approval was granted by the Ethics Committee of the Smith College School for Social Work (Date: April 30, 2018/Number: 1718-076).

\section{References}

Anastas, J. W. (1999). Research design for social work and the human services (2nd ed.). New York: Columbia University Press.

Anastas, J. W. (2012). From scientism to science: How contemporary epistemology can in form practice research. Clinical Social Work Journal, 40(2), 157-165.

Asen, E., \& Fonagy, P. (2012). Mentalization-based therapeutic interventions for families. Journal of Family Therapy, 34(4), 347-370.

Bass, A. (2003). "E" enactments in psychoanalysis: Another medium, another message. Psychoanalytic Dialogues, 13(5), 657-675.

Bateson, G. (1979). Mind and nature: A necessary unity. Bantam Books.

Benjamin, J. (2014). Beyond doer and done to: an intersubjective view of thirdness. In Relational psychoanalysis (Vol. 4, pp. 91-130). Routledge

Betan, E., Heim, A. K., Conklin, C. Z., \& Westen, D. (2005). Countertransference phenomena and personality pathology in clinical practice: An empirical investigation. American Journal of Psychiatry, 16(5), 890-898.

Bromberg, P. (2011). The shadow of the tsunami and the growth of the relational mind. Routledge.
Charmaz, K. (2006). Constructing grounded theory: A practical guide through qualitative analysis. Sage.

Chused, J. F. (2003). The role of enactments. Psychoanalytic Dialogues, 13(5), 677-687.

Cozolino, L. (2017). The neuroscience of psychotherapy: Healing the social brain. W.W. Norton \& Company.

Czander, W. M. (1993). The psychodynamics of work and organizations: Theory and application. The Guilford Press.

Denzin, N. K., \& Lincoln, Y. S. (2011). The SAGE handbook of qualitative research. London: Sage.

Eckstein, R., \& Wallerstein, R. S. (1958). The teaching and learning of psychotherapy. New York: International Universities Press.

Eisenberg, A. (1997). Institutional countertransference: The matrix of social structure and psychic structure. Journal of the American Academy of Psychoanalysis, 25, 237-254.

Figley, C. R. (1995). Compassion fatigue: Toward a new understanding of the costs of caring. In B. H. Stamm (Ed.), Secondary traumatic stress: Self-care issues for clinicians, researchers, and educators (pp. 3-28). The Sidran Press.

Fonagy, P., \& Bateman, A. (2006). Progress in the treatment of borderline personality disorder. British Journal of Psychiatry, 188 , $1-3$.

Gabbard, G. (1989). Splitting in hospital treatment. American Journal of Psychiatry, 146(4), 444-451.

Ginot, E. (2007). Intersubjectivity and neuroscience: Understanding enactments and their therapeutic significance with in emerging paradigms. PsychoanalyticPsychology, 24(2), 317.

Glaser, B., \& Strauss, A. (1967). The discovery of grounded theory. New York: Aldine.

Greene, L. R. (1993). Primitive defenses and the borderline patient's perceptions of the psychiatric treatment team. Psychoanalytic Psychology, 10(4), 533-549.

Guba, E. G., \& Lincoln, Y. S. (1989). Fourth generation evaluation. Sage Publications Inc.

Herrman, H., Trauer, T., \& Warnock, J. (2002). The roles and relationships of psychiatrists and other service providers in mental health services. Australian and New Zealand Journal of Psychiatry, 36(1), 75-80.

Jacobs, T. J. (1986). On countertransference enactments. Journal of the American Psychoanalytic Association, 34(2), 289-307.

Kondrat, M. E. (2013). Person-in-environment. In: Encyclopedia of social work.

Krikorian, S., \& Fowler, J. (2011). A team approach to treatment resistance. In E. M. Plakun (Ed.), Treatment resistance and patient authority: The Austen Riggs Reader (pp. 245-268). WW. Norton \& Company Inc.

Lincoln, Y. S., \& Guba, E. G. (1985). Naturalistic inquiry. Sage Publications Inc.

Main, T. F. (1957). The ailment. British Journal of Medical Psychology, 30(3), 129-145.

Maroda, K. J. (1998). Enactment: When the patient's and analyst's pasts converge. Psychoanalytic Psychology, 15(4), 517-535.

McEwen, B. S. (2007). Physiology and neurobiology of stress and adaptation: Central role of the brain. Physiological Reviews, 87(3), 873-904

Mendelsohn, R. (2012). Parallel process and projective identification in psychoanalytic supervision. The Psychoanalytic Review, 99(3), 297-314.

Menninger, R. W. (1998). Thetherapeutic environment and team approach at the Menninger Hospital. Psychiatry andClinical Neurosciences, 52(S6), 173-176.

Miller, E. (1993). From dependency to autonomy: Studies in organization and change. Free Association Books.

Mitchell, S. (1988). Relational concepts in psychoanalysis. Harvard.

Mitchell, S. A. (1993). Hope and dread in psychoanalysis. Basic Books. 
Mitchell, S. A. (2014). Relationality: From attachment to intersubjectivity. Psychology Press.

Monroe, J., Shay, J., Fisher, L., Makary, C., Rapperport, K., \& Zimering, R. (1995). Preventing compassion fatigue: A team treatment model. In C. R. Figley (Ed.), Compassion fatigue: Coping with secondary traumatic stress disorder in those who treat the traumatized (pp. 209-231). London: Brunner-Routledge.

Morey, C. M. (2019). System enactment: An individual, interpersonal, and organizational perspective. Smith College Studies in Social Work, 89(2), 91-113.

Ogden, T. (1994). Subjects of analysis. Lanham, MD: Rowman and Littlefield.

Pearlman, L. A., \& Saakvitne, K. W. (1995). Countertransference and vicarious traumatization in psychotherapy with incest survivors. New York: W.W. Norton \& Company.

Plakun, E. M. (1997). Enactment and the treatment of abuse survivors. Harvard Review Psychiatry, 5(6), 318-324.

Plakun, E. M. (2007). Perspectives on embodiment: from symptom to enactment and from enactment to sexual misconduct. In J. P. Muller \& J. G. Tillman (Eds.), The embodied subject: Minding the body in psychoanalysis (pp. 103-116). Lanham, MD: Rowman and Littlefield.

Ringstrom, P. A. (2010). Meeting Mitchell's challenge: A comparison of relational psychoanalysis and intersubjective systems theory. Psychoanalytic Dialogues, 20(2), 196-218.

Salhani, D., \& Coulter, I. (2009). The politics of interprofessional working and the struggle for professional autonomy in nursing. Social Science \& Medicine, 68(7), 1221-1228.

Sandler, J. (1976). Countertransference and role-responsiveness. International Review of Psychoanalysis, 3(1), 43-47.

Schore, A. (2012). The science of the art of psychotherapy. W.W. Norton \& Company.

Searles, H. F. (1955). The informational value of the supervisor's emotional experiences. Psychiatry: Journal for the Study of Interpersonal Processes, 18, 135-146.

Shapiro, E. (2011). Examined living: A psychodynamic treatment system for patients with treatment resistant disorders. In E. M. Plakun (Ed.), Treatment resistance and patient authority: The Austen Riggs Reader (pp. 269-284). New York: W.W. Norton \& Company.
Shea, S. E. (2019). Reflective supervision for social work field instructors: Lessons learned from infant mental health. Clinical Social Work Journal, 47(1), 61-71.

Siegel, D. J. (2003). An interpersonal neurobiology of psychotherapy: The developing mind and the resolution of trauma. In M. F. Solomon \& D. J. Siegel (Eds.), Healing trauma: Attachment, mind, body and brain (pp. 1-56). New York: W.W. Norton \& Company.

Siegel, D. J. (2007). The mindful brain: Reflection and attunement in the cultivation of well-being. W.W. Norton \& Company.

Skogstad, W. (2006). Action and thought: Inpatient treatment of severe personality disorders within a psychotherapeutic milieu. In C. Newrith, C. Meux, \& P. Taylor (Eds.), Personality disorder and serious off ending: Hospital treatment models (pp. 161-169). Boca Raton, FL: CRC Press.

Stanton, A. H., \& Schwartz, M. S. (1954). The mental hospital: A study of institutional participation in psychiatric illness and treatment. New York: Basic Books.

Stapley, L. (2001). A large system intervention: The influence of organizational culture. In L. Gould, L. Stapley, \& M. Stein (Eds.), The systems psychodynamics of organizations: Integrating the group relations approach, psychoanalytic and open systems perspectives (pp. 157-173). New York: Karnac.

Strauss, A., \& Corbin, J. (1990). Basics of qualitative research: Grounded theory procedures and techniques. Sage.

Strauss, A., \& Corbin, J. (1998). Basics of qualitative research: Techniques and procedures for developing grounded theory. Thousand Oaks, CA: Sage.

van der Kolk, B. (2014). The body keeps the score. Penguin Books.

von Bertalanffy, L. (1969). General systems theory: Foundations, development, applications. George Braziller Inc.

Publisher's Note Springer Nature remains neutral with regard to jurisdictional claims in published maps and institutional affiliations.

Cathleen M. Morey PhD, LICSW is the Director of Clinical Social Work at the Austen Riggs Center. She is adjunct faculty at the Smith College School for Social Work and the University of Connecticut School of Social Work. She maintains a private practice in Stockbridge, MA focusing on psychotherapy and consultation. 